Check for updates

Cite this: J. Mater. Chem. C, 2020, 8, 11540

Received 18th May 2020,

Accepted 16th July 2020

DOI: $10.1039 / \mathrm{d} 0 \mathrm{tc02381g}$

rsc.li/materials-c

\title{
Supramolecular oligourethane gel as a highly selective fluorescent "on-off-on" sensor for ions $\uparrow$
}

\author{
Yulin Feng, $\ddagger^{a}$ Nan Jiang, $\ddagger^{a}$ Dongxia Zhu, (D) *a Zhongmin Su (D) $\star^{a}$ and \\ Martin R. Bryce (D) *b
}

\begin{abstract}
Stimuli-responsive supramolecular gels (SRSGs) are an important class of smart materials. It is of practical importance to develop an SRSG which can both detect and remove toxic metal ions. We have designed and synthesized an aggregation induced emission (AIE)-active oligourethane (OU) gelator which self-assembles into a supramolecular gel (OUG), through hydrogen-bonding, $\pi-\pi$ stacking and van der Waals interactions. By taking advantage of the weak and dynamic nature of these non-covalent bonds, OUG shows stimuli-response to multiple factors. Importantly, OUG has the capacity for real-time detection and high selectivity for $\mathrm{Fe}^{3+}, \mathrm{HSO}_{4}{ }^{-}$and $\mathrm{F}^{-}$. The lowest detection limits are in the range of $5.89 \times 10^{-9}$ to $8.17 \times 10^{-8} \mathrm{M}$, indicating high sensitivity. More importantly, OUG is shown to adsorb and separate $\mathrm{Fe}^{3+}$ from aqueous solution, with an absorbing rate of up $97.5 \%$. A simple writing board was fabricated, which could be written repeatedly and reused. OUG acts as a reversible and recyclable "on-off-on" fluorescence sensor via competitive cation- $\pi$ and cation-anion interactions. OUG has great potential as an environmentally sustainable probe for ions.
\end{abstract}

\section{Introduction}

Stimuli-responsive supramolecular gels (SRSGs) have the ability to respond to a chemical substance, ${ }^{1}$ light, ${ }^{2}$ heat ${ }^{3} \mathrm{pH}^{4}$ or pressure. ${ }^{5}$ They have been applied in chemical sensors, ${ }^{6}$ displays, ${ }^{7}$ drug deliveries ${ }^{8}$ and other fields. ${ }^{9}$ Responsive behavior can be achieved by a gel-sol state transition or by changing the luminescence. ${ }^{7,10}$ The latter response works by changing the gel's fluorescence intensity or color, and can be free from the influence of temperature, ${ }^{2} \mathrm{pH}^{11}{ }^{1}$ an oxidizing agent, ${ }^{8}$ and other factors. ${ }^{12}$ Therefore luminescence detection has considerably higher sensitivity and more reliable real-time response. ${ }^{13-15}$ Traditional conjugated gelators usually suffer from aggregationcaused quenching (ACQ), which sharply weakens the emission behavior in aggregation or solid states, thereby limiting their applications. ${ }^{16}$ The emergence of polymers/oligomers with aggregation-induced emission (AIE) properties has been a

\footnotetext{
${ }^{a}$ Key Laboratory of Nanobiosensing and Nanobioanalysis at Universities of Jilin Province, Department of Chemistry, Northeast Normal University, 5268 Renmin Street, Changchun, Jilin Province 130024, P. R. China. E-mail: zhudx047@nenu.edu.cn, zmsu@nenu.edu.cn

${ }^{b}$ Department of Chemistry, Durham University, Durham, DH1 3LE, UK.

E-mail:m.r.bryce@durham.ac.uk

$\dagger$ Electronic supplementary information (ESI) available: ${ }^{1} \mathrm{H}$ NMR spectra and photophysical properties. See DOI: 10.1039/d0tc02381g

\$ These authors contributed equally to the preparation of this work.
}

breakthrough in the field. ${ }^{17,18}$ In addition to their excellent emission characteristics, AIE-active supramolecular gels show strong absorption activity and synergistic effects because of their large contact area with analytes. ${ }^{19}$

Recently, our group has explored AIE-active poly/oligourethane-based unconventional luminophores, which are without typical polycyclic $\pi$-conjugated units. These materials show obvious advantages like environmental friendliness, excellent hydrophilicity, chain flexibility, ease of synthesis and structural versatility compared with traditional organic luminescent materials. ${ }^{20-22}$

$\mathrm{Fe}^{3+}$ is an indispensable element in the process of oxygen uptake and metabolism. ${ }^{23}$ However, an excess of $\mathrm{Fe}^{3+}$ might cause pathological diseases like cancer and organ dysfunction. ${ }^{24}$ $\mathrm{F}^{-}$and $\mathrm{HSO}_{4}{ }^{-}$also play essential roles in human biological processes, ${ }^{25,26}$ although undue fluoride may cause kidney problems, dental and skeletal fluorosis. ${ }^{27} \mathrm{HSO}_{4}{ }^{-}$can produce poisonous $\mathrm{SO}_{4}{ }^{2-}$ under acidic conditions, which will stimulate the skin and eyes and can even cause respiratory paralysis. ${ }^{28}$ Thus, methods to efficiently detect these ions have received extensive attention. The established detection techniques, such as inductively coupled plasma spectroscopy, ${ }^{29}$ high performance liquid chromatography (HPLC) ${ }^{30}$ and electrochemical methods, ${ }^{31}$ all require tedious sample preparations, sophisticated instruments and professional operators. However, fluorescent sensor molecules, which convert and amplify the signals into a visible 
and easily recognized fluorescent output, offer a more significant practical method. ${ }^{6,32}$

Herein, we report an AIE-active supramolecular oligourethane gel (OUG) and demonstrate its usage as a specific $\mathrm{Fe}^{3+}$ sensor in an aqueous environment. The material is based on the following design criteria: (i) inserting benzophenone into an oligourethane (OU) backbone provides $\mathrm{C}=\mathrm{O}$ units with prominent hydrogen-bonding sites for self-assembly, and formation of oxygen clusters, which could enhance fluorescent emission. $^{33}$ (ii) Inserting linear 1,6-diisocyanatohexane offers strong van der Waals interactions among alkyl chains, limiting internal rotation of the molecular chains, thereby blocking the non-radiative pathways and favoring AIE. Taking advantage of the rich hydrogen bond acceptors/donors $(\mathrm{C}=\mathrm{O} / \mathrm{N}-\mathrm{H})$ among the oligourethane skeleton, ${ }^{34-41}$ we introduced solvents with hydrogen-bonding acceptor units $(\mathrm{C}=\mathrm{O}$ or $\mathrm{S}=\mathrm{O})$ as external crosslinking agents, to self-assemble a supramolecular oligourethane gel (OUG) relying on multiple hydrogen bonds.

\section{Results and discussion}

\section{Synthesis and characterization}

The OU was synthesized through a facile procedure as shown in Scheme S1 (ESI $\dagger$ ), by the reaction of $4,4^{\prime}$-dihydroxybenzophenone, hexamethylene diisocyanate and DABCO in anhydrous tetrahydrofuran and end-capping with polyethylene glycol monomethyl ether to give a viscous solution. The product was purified by a counter precipitation method. ${ }^{1} \mathrm{H}$ NMR and FTIR characterization data are given in Fig. 1a and in the ESI, $\dagger$ confirming the structure of OU. The $M_{\mathrm{n}}$ value of $1814 \mathrm{~g} \mathrm{~mol}^{-1}$, calculated from the

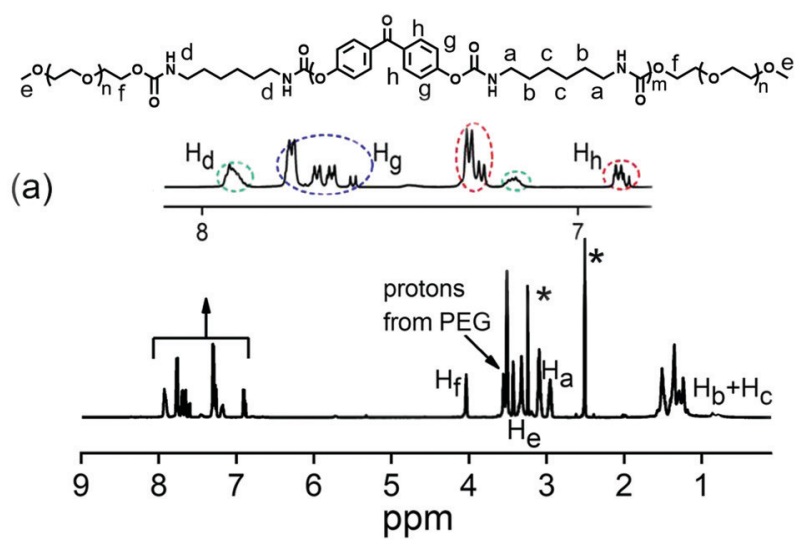

(b)

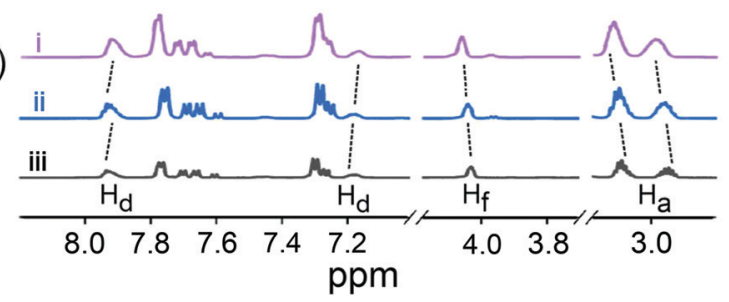

Fig. 1 (a) ${ }^{1} \mathrm{H}$ NMR spectrum of $\mathrm{OU}$ in DMSO- $d_{6}$ (* indicates peaks from the solvent and water); $\mathrm{H}$-atom labeling is shown on the structure of $\mathrm{OU}$. (b) The partial ${ }^{1} \mathrm{H}$ NMR spectra of OU in DMSO- $d_{6}$ at different concentrations: (i) $0.925 \mathrm{mM}$, (ii) $3.7 \mathrm{mM}$, and (iii) $25.0 \mathrm{mM}$.
${ }^{1} \mathrm{H}$ NMR data, established that OU should be classified as an oligomer. $^{42}$ The FTIR spectra (Fig. S1, ESI $\dagger$ ) showed absorbance bands at $3323 \mathrm{~cm}^{-1}$ and $1706 \mathrm{~cm}^{-1}$, assigned to stretching vibrations of $\mathrm{N}-\mathrm{H}$ and $\mathrm{C}=\mathrm{O}$, indicating the formation of amide bonds. Absorbance bands at $2936 \mathrm{~cm}^{-1}$ and $2860 \mathrm{~cm}^{-1}$ correspond to $v\left(-\mathrm{CH}_{2}-\right)$ and at $1163 \mathrm{~cm}^{-1}$ correspond to $v(\mathrm{C}-\mathrm{O}-\mathrm{C})$ stretching vibrations. The UV-Vis absorption spectrum of OU in the solid-state (Fig. S2, ESI $\dagger$ ) showed a major peak at $277 \mathrm{~nm}$ from a $\pi-\pi^{*}$ transition of the aromatic rings. ${ }^{43}$

\section{Self-assembly gelation}

OU spontaneously self-assembles in certain solvents (notably dimethyl formamide and dimethylsulfoxide) transforming into a supramolecular gel (Table S1, ESI $\dagger$ ). The lowest critical gelation concentration (CGC) of OU is $4 \%\left(\mathrm{w} / \mathrm{v}, 10 \mathrm{mg} \mathrm{mL}^{-1}=\right.$ $1 \%)$, and the corresponding gel-sol transition temperature $\left(T_{\text {gel }}\right)$ is $85-87{ }^{\circ} \mathrm{C}$. In order to gain an insight into the selfassembly mechanism, ${ }^{1} \mathrm{H}$ NMR, FTIR, XRD and urea addition experiments were conducted. ${ }^{1} \mathrm{H}$ NMR spectra were recorded for different concentrations of OU in DMSO- $d_{6}$ (Fig. 1b). The $\mathrm{H}_{\mathrm{a}}$ and $\mathrm{H}_{\mathrm{f}}$ proton signals are shifted $c a .0 .04$ and 0.03 ppm upfield compared to pure OUG upon adding $25 \mathrm{mM} \mathrm{Fe}^{3+}$. Meanwhile, the signals of protons $\mathrm{H}_{\mathrm{d}}$ (the $\mathrm{NH}$ groups) shifted slightly downfield ca. 0.01 ppm. $^{44}$ These results confirmed the $\mathrm{H}$-bonding interactions between amide groups and van der Waals interactions between alkyl chains. Comparing the FTIR data before and after gelation (Fig. S1, ESI $\dagger$ ), the $\mathrm{N}-\mathrm{H}$ stretching absorbance bands of OUG are broader and move to significantly higher wavenumbers (3323 to $3361 \mathrm{~cm}^{-1}$ ) in the solid state compared to the gel state: these data suggest hydrogen bonds play a critical role in the gelation process. ${ }^{45,46}$

It is well known that adding urea, which has a high propensity to form hydrogen bonds, can disrupt existing hydrogen bonds in a supramolecular structure. ${ }^{47,48}$ Accordingly, adding urea (10 equiv.) into OUG and heating the gel, led to the formation of a sol. It was observed that after adding urea, the sol did not revert back to gel, even when the OUG-urea mixture was cooled at $15{ }^{\circ} \mathrm{C}$ for several days, indicating that the gelation is driven by hydrogen bonds among OU molecular chains (Fig. 2a). Besides, the X-ray diffraction (XRD) peaks of OUG

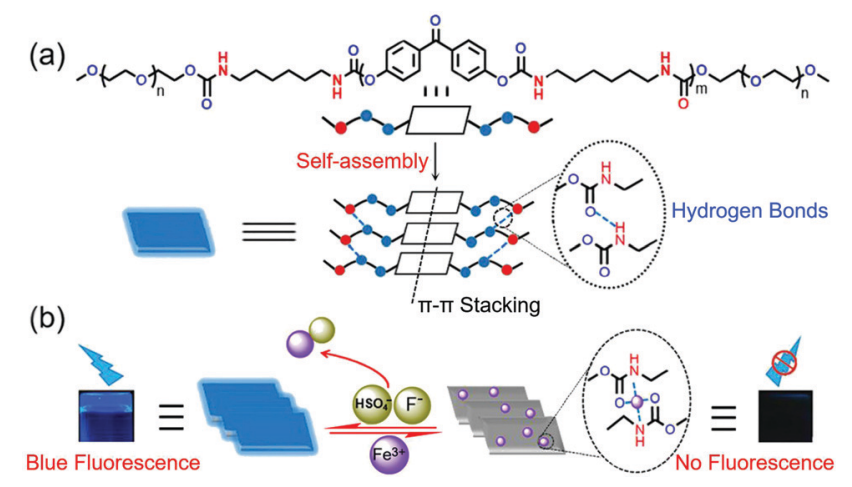

Fig. 2 (a) Structure of $\mathrm{OU}$ and its self-assembly gelation process. (b) Schematic diagram for the ion sensing processes of OUG 
$2 \theta=20.54^{\circ}, 23.22^{\circ}$ corresponding to $d$-spacings of $4.32 \AA$ and $3.83 \AA$, respectively, also indicated the presence of $\pi-\pi$ stacking interactions (Fig. S11b, ESI $\dagger$ ), further promoting the selfassembly behavior. OUG showed weak fluorescence in the sol state, however, after transforming to the gel state, the emission intensity of OUG at $439 \mathrm{~nm}$ increased 6 times (Fig. S3, ESI $\dagger$ ), indicating that $\mathrm{OU}$ is an AIE-active gelator. ${ }^{49}$

\section{Stimuli-responsive behaviors}

OUG exhibits a high selectivity to $\mathrm{Fe}^{3+}$ over other metal ions. By monitoring the change of fluorescence, we investigated the recognition characteristics of OUG towards metal ions. Using nitrate salts as the cation sources, an aqueous metal ion solution of $\mathrm{Na}^{+}, \mathrm{Ca}^{2+}, \mathrm{Co}^{2+}, \mathrm{Cu}^{2+}, \mathrm{Mn}^{2+}, \mathrm{Ni}^{2+}, \mathrm{Cr}^{3+}, \mathrm{La}^{3+}, \mathrm{Fe}^{3+}$, $\mathrm{Sr}^{2+}, \mathrm{Ce}^{3+}, \mathrm{Ag}^{+}, \mathrm{Al}^{3+}, \mathrm{Mg}^{2+}, \mathrm{Cd}^{2+}, \mathrm{Pb}^{2+}$ or $\mathrm{Fe}^{2+}(c=0.2 \mathrm{M})$ was added to the OUG to generate the corresponding metal-gels. ${ }^{50-53}$ As shown in Fig. 3a and c, initially, the OUG had a strong blue fluorescence emission. When different metal ions were added, only $\mathrm{Fe}^{3+}$ quenched the fluorescence of OUG. Thus, the OUG could effectively and selectively detect $\mathrm{Fe}^{3+}$. To further evaluate the sensitivity of OUG for $\mathrm{Fe}^{3+}$, the fluorescence behavior of OUG was monitored by continuous titrations with $\mathrm{Fe}^{3+}$. As shown in Fig. S5a (ESI $\dagger$ ), with the increasing addition of $\mathrm{Fe}^{3+}(0-1.1$ equiv.), the emission intensity of the corresponding metal-gels (OUFeG) at $439 \mathrm{~nm}$ gradually decreased. The limit of detection (LOD) of OUG towards $\mathrm{Fe}^{3+}$ was calculated to be $5.89 \times 10^{-9} \mathrm{M}$ based on the $3 \delta / S$ method $^{54}$ (Fig. S4 and S5a, ESI $\dagger$ ), confirming the high selectivity of OUG as a sensor for $\mathrm{Fe}^{3+}$ compared with other reported sensor
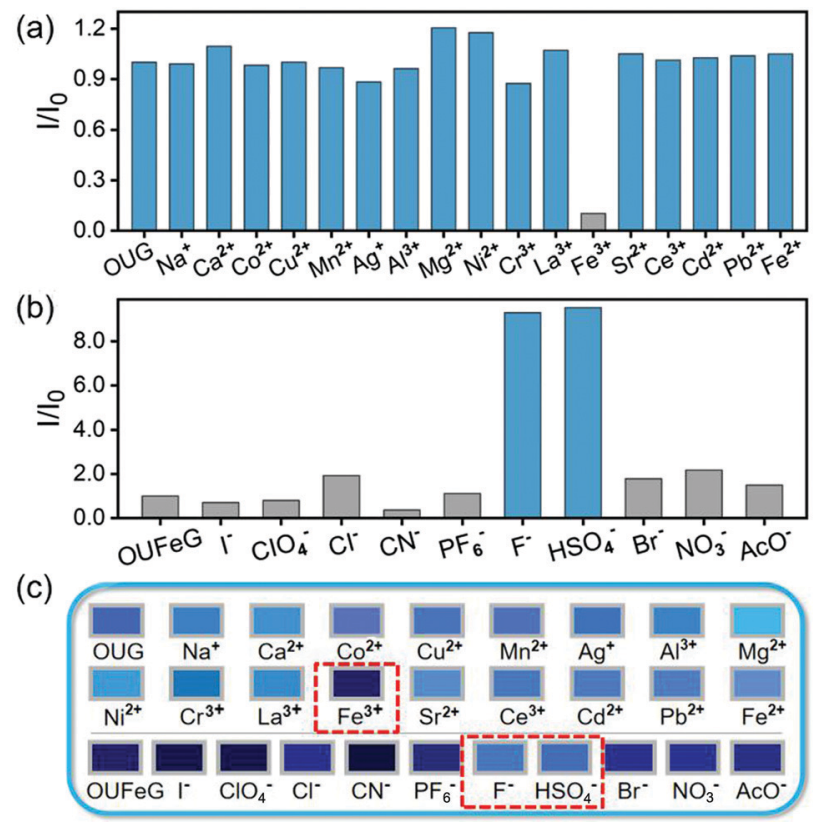

Fig. 3 (a) Fluorescence responses of OUG (in DMF, 10\% (w/v)) toward $\mathrm{Fe}^{3+}$ and other metal ions at room temperature. (b) Fluorescence responses of OUG (in DMF, 10\% (w/v)) toward $\mathrm{F}^{-}, \mathrm{HSO}_{4}{ }^{-}$and other anions at room temperature. All data represent the fluorescence intensity ratio $/ / I_{0}$ ( $/$ and $I_{0}$ are the final fluorescence intensity and initial fluorescence intensity for the gel with each ion tested at $384 \mathrm{~nm}$ ). (c) Photos of OUG fluorescence responses to various cations and anions. systems (Table S2, ESI $\dagger$ ). The high selectivity of OUG to $\mathrm{Fe}^{3+}$ is attributed to two reasons: firstly, unpaired electrons in $\mathrm{Fe}^{3+}$ cause a paramagnetic effect, prompting energy dissipation of excited states through non-radiative pathways. ${ }^{55}$ Secondly, the high ionic strength of $\mathrm{Fe}^{3+}$ could easily induce the transfer of $\pi$-electrons from the urethane backbone to $\mathrm{Fe}^{3+}$ through cation $-\pi$ interactions. ${ }^{56}$ both of these effects will cause the fluorescence quenching of OUG.

The response of OUFeG towards anions was further investigated, using tetrabutylammonium salts as the anion sources, adding aqueous solutions of anions including $\mathrm{HSO}_{4}{ }^{-}, \mathrm{ClO}_{4}{ }^{-}$, $\mathrm{I}^{-}, \mathrm{CN}^{-}, \mathrm{PF}_{6}^{-}, \mathrm{F}^{-}, \mathrm{Cl}^{-}, \mathrm{Br}^{-}, \mathrm{NO}_{3}{ }^{-}, \mathrm{AcO}^{-}(c=0.2 \mathrm{M})$ to OUFeG. ${ }^{57-60}$ As shown in Fig. $3 \mathrm{~b}$ and $\mathrm{c}$, when $\mathrm{HSO}_{4}{ }^{-}$and $\mathrm{F}^{-}$ were added the emission at $439 \mathrm{~nm}$ recovered immediately, whereas the other anions did not lead to a comparable fluorescence recovery of OUFeG. These phenomena confirmed the excellent selectivity of OUFeG towards $\mathrm{HSO}_{4}{ }^{-}$and $\mathrm{F}^{-}$. This selectivity is explained by the strong coordinating capacity of $\mathrm{HSO}_{4}{ }^{-}$and $\mathrm{F}^{-}$with $\mathrm{Fe}^{3+}$, in contrast to (metal-free) OUG. These two anions could competitively bind to $\mathrm{Fe}^{3+}$ in OUFeG and induce the re-emission of OUG. Titration experiments evaluated the sensing efficiency of OUFeG to $\mathrm{HSO}_{4}{ }^{-}$and $\mathrm{F}^{-}$. Limit of detection (LOD) values were calculated according to the $3 \delta / S$ method to be $8.17 \times 10^{-8} \mathrm{M}$ for $\mathrm{F}^{-}$and $1.16 \times 10^{-8} \mathrm{M}$ for $\mathrm{HSO}_{4}{ }^{-}$(Fig. S5b and Fig. S7-S9, ESI $\dagger$ ). Notably the LOD values of OUFeG are lower than many other reported $\mathrm{F}^{-}$and $\mathrm{HSO}_{4}{ }^{-}$ fluorescence sensors (Table S2, ESI $\dagger$ ). The gel OUG selectively senses $\mathrm{Fe}^{3+}$, and the metal-gel OUFeG selectively responds to $\mathrm{F}^{-}$and $\mathrm{HSO}_{4}{ }^{-}$. This property makes gel OUG and metal-gel OUFeG act as an efficient "on-off-on" fluorescence sensor controlled by $\mathrm{Fe}^{3+}, \mathrm{HSO}_{4}{ }^{-}$or $\mathrm{F}^{-}$with excellent reversibility. A simple regeneration treatment verified the recyclability of OUG. An anion solution $\left(\mathrm{F}^{-}\right.$or $\mathrm{HSO}_{4}{ }^{-}, 2 \times 10^{-5} \mathrm{~mol} \mathrm{~L}^{-1}$; $10 \mathrm{~mL}$ ) was added into metal-gel OUFeG, stirring the mixture for $5 \mathrm{~min}$, centrifuging and recycling OUG for again detecting ions. As shown in Fig. S10 (ESI $\dagger$ ), after five consecutive cycles, the intensity of the OUG signal is essentially unchanged, indicating the excellent recyclability and reversibility of the OUG for the detection of $\mathrm{Fe}^{3+}$ and $\mathrm{HSO}_{4}{ }^{-}$or $\mathrm{F}^{-}$.

\section{Mechanism of cation-anion sensor}

When adding aqueous solution of $\mathrm{Fe}^{3+}$ into OUG, $\mathrm{Fe}^{3+}$ can diffuse in the solution. Due to the high ionic strength of $\mathrm{Fe}^{3+}$, the $\pi$-electrons in the urethane groups of OUG are easily induced to form cation $-\pi$ interactions quenching the fluorescence. The recognition mechanisms of OUG to $\mathrm{Fe}^{3+}$ were investigated via a combination of ${ }^{1} \mathrm{H}$ NMR, FTIR, XRD and SEM analysis. In the ${ }^{1} \mathrm{H}$ NMR titration experiments, with the increasing amount of $\mathrm{Fe}^{3+}$ added into OU, protons $\mathrm{H}_{\mathrm{d}}, \mathrm{H}_{\mathrm{f}}$ and $\mathrm{H}_{\mathrm{a}}$ all displayed clear upfield shifts (Fig. 4a), indicating that the OUG combined with $\mathrm{Fe}^{3+}$ via cation $-\pi$ interactions between the urethane groups and $\mathrm{Fe}^{3+} \cdot{ }^{56,61}$ As Fig. $4 \mathrm{~b}$ shows, by adding $\mathrm{F}^{-}$ or $\mathrm{HSO}_{4}{ }^{-}(3.7 \mathrm{mM})$ into a sample of $\mathrm{OU}$ (with 2 equiv. $\mathrm{Fe}^{3+}$ ), all the protons recovered the chemical shifts as in the original OU: $\mathrm{H}_{\mathrm{d}}$ (from 7.85 to $7.93 \mathrm{ppm}$ and 7.09 to $7.19 \mathrm{ppm}$ ), $\mathrm{H}_{\mathrm{f}}$ (from 3.94 to $4.03 \mathrm{ppm}$ ) and $\mathrm{H}_{\mathrm{a}}$ (from 3.01 to $3.09 \mathrm{ppm}$ and 2.87 to 


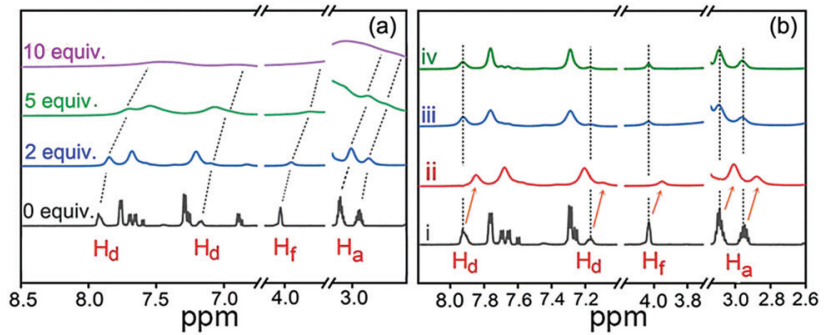

Fig. 4 (a) ${ }^{1} \mathrm{H}$ NMR titration spectra (298 K) of $0.925 \mathrm{mM} \mathrm{OU}$ with various equivalents of $\mathrm{Fe}^{3+}$ in DMSO- $d_{6}$ solution. (b) ${ }^{1} \mathrm{H}$ NMR spectra of (i) $\mathrm{OU}$ (ii) OUFeG (iii) OUFeG $+\mathrm{HSO}_{4}^{-}$and (iv) OUFeG $+\mathrm{F}^{-}$

$2.95 \mathrm{ppm}$ ), which indicated the cation-anion interactions between $\mathrm{Fe}^{3+}$ and $\mathrm{F}^{-}$or $\mathrm{HSO}_{4}{ }^{-}$could release the $\pi$-electrons of urethane groups, thus recovering the fluorescence of OUG.

In the FTIR experiments (Fig. S11a, ESI $\dagger$ ), when $\mathrm{Fe}^{3+}$ was added into OUG to form OUFeG, the stretching absorbance bands of $\mathrm{N}-\mathrm{H}, \mathrm{C}=\mathrm{O}$ and $\mathrm{C}-\mathrm{O}-\mathrm{C}$ shifted from $3361 \mathrm{~cm}^{-1}$, $1708 \mathrm{~cm}^{-1}$ and $1161 \mathrm{~cm}^{-1}$ to $3480 \mathrm{~cm}^{-1}, 1673 \mathrm{~cm}^{-1}$ and $1158 \mathrm{~cm}^{-1}$ respectively, which further confirmed that $\mathrm{Fe}^{3+}$ interacts with $\pi$-electrons of the urethane groups, thus influencing $\mathrm{H}$-bonds between the amide groups. ${ }^{6,57}$ After the addition of $\mathrm{F}^{-}$or $\mathrm{HSO}_{4}{ }^{-}$into the OUFeG, the $\mathrm{C}=\mathrm{O}, \mathrm{N}-\mathrm{H}$ and $\mathrm{C}-\mathrm{O}-\mathrm{C}$ all reverted to their initial positions (Fig. S11a, ESI $\dagger$ ). These observations suggested that $\mathrm{F}^{-}$and $\mathrm{HSO}_{4}{ }^{-}$competitively bound to $\mathrm{Fe}^{3+}$ rather than to OUG. Moreover, the XRD peaks of OUG moved with adding $\mathrm{Fe}^{3+}$ into OUG, and recovered when $\mathrm{F}^{-}$or $\mathrm{HSO}_{4}{ }^{-}$was added into OUFeG (Fig. S11b, ESI $\dagger$ ).

To get further insight into the mechanism of cation-anion sensing, as shown in Fig. 5, the SEM studies were carried out. Fig. 5a demonstrates that gel OUG shows a lamellar stacking structure with a smooth surface. This structure was converted into a honeycomb structure in the metal-gel OUFeG (Fig. 5b), while in gel OUFeG $+\mathrm{HSO}_{4}{ }^{-}$and $\mathbf{O U F e G}+\mathrm{F}^{-}$, the image again showed a smooth lamellar stacking structure (Fig. $5 \mathrm{c}$ and d). Such morphological change is attributed to the cation- $\pi$ interactions between OUG and $\mathrm{Fe}^{3+}$, breaking hydrogen bonding
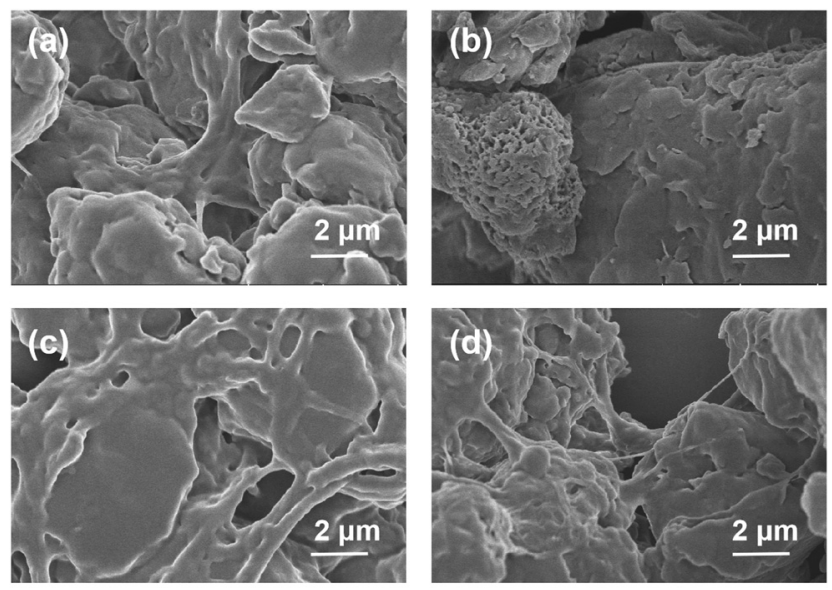

Fig. 5 SEM images of (a) OUG; (b) OUFeG; (c) OUFeG $+\mathrm{HSO}_{4}^{-}$ (d) OUFeG $+F^{-}$. between the OUG chains and modifying the supramolecular structure. ${ }^{57,61}$ After adding $\mathrm{F}^{-}$or $\mathrm{HSO}_{4}{ }^{-}$into OUFeG, $\pi$-electrons of OUG were released, hydrogen bonds are rebuilt and the morphology is recovered. These experimental results indicated that the fluorescence of OUG can be reversibly switched by $\mathrm{Fe}^{3+}$ (off) and then by $\mathrm{F}^{-}$or $\mathrm{HSO}_{4}{ }^{-}$(on), through repeated competition between cation- $\pi$ and cation-anion interactions (Fig. $2 b$ ).

\section{Application in the rapid removal of $\mathrm{Fe}^{3+}$}

The development of new sorbents for the sensing and extraction of metal ions from environmental and biological samples is of current importance. ${ }^{62,63}$ The performance of OUG to effectively remove $\mathrm{Fe}^{3+}$ from aqueous solution was analyzed by atomic absorption spectrometry (Table S3, ESI $\dagger$ ). Specifically, OUG $(0.2 \mathrm{~g})$ was added to a dilute aqueous solution of $\mathrm{Fe}^{3+}$ $\left(1 \times 10^{-5} \mathrm{~mol} \mathrm{~L}^{-1}\right.$ in $10 \mathrm{~mL}$ water $)$ and the mixture was stirred for $10 \mathrm{~min}$. The precipitate was separated by centrifuging at $5000 \mathrm{rpm}$ for $5 \mathrm{~min}$ and the supernatant was retained, the residual concentration of $\mathrm{Fe}^{3+}$ was about $2.5 \times 10^{-7} \mathrm{~mol} \mathrm{~L}^{-1}$. Experimental results demonstrated that $97.5 \%$ of $\mathrm{Fe}^{3+}$ could be efficiently removed by OUG even from extremely dilute solutions, which indicated the excellent adsorption capacity of OUG towards $\mathrm{Fe}^{3+}{ }^{3,56,57,64}$ As shown in Fig. S6 (ESI $\dagger$ ), adsorption tests of OUG toward $\mathrm{Fe}^{3+}$ in the $\mathrm{pH}$ range from 4 to 10 showed that the adsorption of OUG toward $\mathrm{Fe}^{3+}$ showed excellent stability at different $\mathrm{pH}$ conditions (in the range of 92.1-97.5\%). OUG can selectively detect with high-efficiency and rapidly remove toxic $\mathrm{Fe}^{3+}$, offering potential practical applications in combating heavy metal ion pollution and in environmental remediation.

\section{Application as a writing display material}

Based on the above-mentioned "on-off-on" properties, the OUG has a great potential as a rewritable fluorescent display material. As a proof-of-concept a rewritable board was constructed (Fig. 6). The detailed steps are described as follows: (i) OUG sol (10\%) was poured onto a clean quartz plate surface and dried under ambient conditions to give a film emitting strong blue fluorescence under ultraviolet radiation (365 nm). (ii) Writing the symbol "Fe" on the film with a brush dipped in

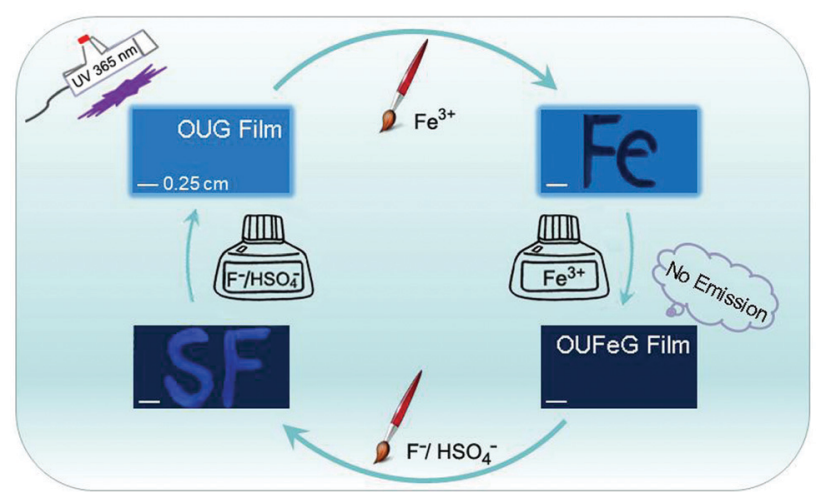

Fig. 6 An erasable writing board of OUG based on its ion-controlled fluorescent switching properties. 
aqueous $\mathrm{Fe}^{3+}$ solution $(0.3 \mathrm{M})$, a dark "Fe" image was clearly displayed due to the fluorescence quenching effect of $\mathrm{Fe}^{3+}$ on OUG. (iii) The whole OUG film was transformed into a nonfluorescent display board by brushing with $\mathrm{Fe}^{3+}$ solution. (iv) Two new letters "S" and "F" could be written again with the same brushing method using $\mathrm{HSO}_{4}{ }^{-}$and $\mathrm{F}^{-}$solutions $(0.3 \mathrm{M})$, respectively. Visually, the letters emitted blue fluorescence under a UV lamp. Combining these practically very simple processes with the excellent recyclability of OUG (discussed above; Fig. S10, ESI $\dagger$ ) means that OUG has promising applications as a fluorescent writing display material.

\section{Conclusion}

In conclusion, a novel supramolecular AIE gel, OUG, was designed and synthesized by a straightforward "one-pot" procedure. The dynamic and reversible noncovalent interactions endow OUG with distinct advantages of a reversible and highly sensitive response to $\mathrm{Fe}^{3+}, \mathrm{HSO}_{4}{ }^{-}$, and $\mathrm{F}^{-}$, acting as an "onoff-on" fluorescent sensor for these cationic and anionic species. Importantly, OUG can absorb up to $97.5 \% \mathrm{Fe}^{3+}$ from a water environment. This rapid, simple, low cost and highly sensitive material has great potential for practical applications in intelligent sensing, handling heavy metal ion pollution and environmental remediation.

\section{Conflicts of interest}

There are no conflicts to declare.

\section{Acknowledgements}

The work was funded by NSFC (No. 51473028), the key scientific and technological project of Jilin province (20150204011GX, 20160307016GX, 20190701010GH), the Development and Reform Commission of Jilin province (20160058, 2020C035-5), Open Project of State Key Laboratory of Supramolecular Structure and Materials (sklssm202039). M. R. B. thanks EPSRC grant EL/L02621X/1 for funding.

\section{Notes and references}

1 L.-J. Chen, G.-Z. Zhao, B. Jiang, B. Sun, M. Wang, L. Xu, J. He, Z. Abliz, H. Tan, X. Li and H.-B. Yang, J. Am. Chem. Soc., 2014, 136, 5993-6001.

2 S.-H. Hsiao and S.-h. Hsu, ACS Appl. Mater. Interfaces, 2018, 10, 29273-29287.

3 J. B. Beck and S. J. Rowan, J. Am. Chem. Soc., 2003, 125, 13922-13923.

4 M. Zhang, D. Xu, X. Yan, J. Chen, S. Dong, B. Zheng and F. Huang, Angew. Chem. Int. Ed., 2012, 51, 7011-7015.

5 J. Lai, H. Zhou, Z. Jin, S. Li, H. Liu, X. Jin, C. Luo, A. Ma and W. Chen, ACS Appl. Mater. Interfaces, 2019, 11, 26412-26420.
6 Y.-M. Zhang, W. Zhu, W.-J. Qu, K.-P. Zhong, X.-P. Chen, H. Yao, T.-B. Wei and Q. Lin, Chem. Commun., 2018, 54, 4549-4552.

7 Q. Lin, B. Sun, Q.-P. Yang, Y.-P. Fu, X. Zhu, Y.-M. Zhang and T.-B. Wei, Chem. Commun., 2014, 50, 10669-10671.

8 X. Cheng, Y. Jin, T. Sun, R. Qi, H. Li and W. Fan, Colloids Surf., B, 2016, 141, 44-52.

9 M. Ikeda, T. Tanida, T. Yoshii, K. Kurotani, S. Onogi, K. Urayama and I. Hamachi, Nat. Chem., 2014, 6, 511-518.

10 Z.-Y. Li, Y. Zhang, C.-W. Zhang, L.-J. Chen, C. Wang, H. Tan, Y. Yu, X. Li and H.-B. Yang, J. Am. Chem. Soc., 2014, 136, 8577-8589.

11 K. Manokruang and D. S. Lee, Macromol. Biosci., 2013, 13, 1195-1203.

12 T. Tanaka, Phys. Rev. Lett., 1978, 40, 820-823.

13 Y. Chang, J. Fu, K. Yao, B. Li, K. Xu and X. Pang, Dyes Pigm., 2019, 161, 331-340.

14 B.-J. Wang, W.-K. Dong, Y. Zhang and S. F. Akogun, Sens. Actuators, B, 2017, 247, 254-264.

15 X. Liu, T. Chen, F. Yu, Y. Shang, X. Meng and Z.-R. Chen, Macromolecules, 2020, 53, 1224-1232.

16 C.-W. Zhang, B. Ou, S.-T. Jiang, G.-Q. Yin, L.-J. Chen, L. Xu, X. Li and H.-B. Yang, Polym. Chem., 2018, 9, 2021-2030.

17 P. Q. Nhien, W.-L. Chou, T. T. K. Cuc, T. M. Khang, C.-H. Wu, N. Thirumalaivasan, B. T. B. Hue, J. I. Wu, S. P. Wu and H.-C. Lin, ACS Appl. Mater. Interfaces, 2020, 12, 10959-10972.

18 Y. Bao, E. Guégain, V. Nicolas and J. Nicolas, Chem. Commun., 2017, 53, 4489.

19 R. Hu, A. Qin and B. Z. Tang, Prog. Polym. Sci., 2020, 100, 101176.

20 N. Jiang, G.-F. Li, B.-H. Zhang, D.-X. Zhu, Z.-M. Su and M. R. Bryce, Macromolecules, 2018, 51, 4178-4184.

21 N. Jiang, G. Li, W. Che, D. Zhu, Z. Su and M. R. Bryce, J. Mater. Chem. C, 2018, 6, 11287-11291.

22 N. Jiang, D. Zhu, Z. Su and M. R. Bryce, J. Mater. Chem. C, 2020, 8, 5137-5142.

23 K. P. Carter, A. M. Young and A. E. Plamer, Chem. Rev., 2014, 114, 4564-4601.

24 Y. Fang, J. Tan, H. Choi, S. Lim and D.-H. Kim, Sens. Actuators, B, 2018, 259, 155-161.

25 Y. Li, Y. Duan, J. Zheng, J. Li, W. Zhao, S. Yang and R. Yang, Anal. Chem., 2013, 85, 11456-11463.

26 U. Haldar and H.-i. Lee, Polym. Chem., 2018, 9, 4882-4890. 27 L. Li, Y. Ji and X. Tang, Anal. Chem., 2014, 86, 10006-10009. 28 H. J. Kim, S. Bhuniya, R. K. Mahajan, R. Puri, H. Liu, K. C. Ko, J. Y. Lee and J. S. Kim, Chem. Commun., 2009, 7128-7130.

29 M. Türkmen, A. Türkmen, Y. Tepe, Y. Töre and A. Ateş, Food Chem., 2009, 113, 233-237.

30 V. Fernández and G. Winkelmann, Biometals, 2005, 18, 53-62.

31 B. Bansod, T. Kumar, R. Thakur, S. Rana and I. Singh, Biosens. Bioelectron., 2017, 94, 443-455.

32 X.-Y. Xu and B. Yan, ACS Appl. Mater. Interfaces, 2015, 7, 721-729. 
33 Q. Zhou, B. Cao, C. Zhu, S. Xu, Y. Gong, W. Z. Yuan and Y. Zhang, Small, 2016, 12, 6586-6592.

34 D. Zhang, Y. Zhang, Y. Fan, M.-N. Rager, V. Guérineau, L. Bouteiller, M.-H. Li and C. M. Thomas, Macromolecules, 2019, 52, 2719-2724.

35 K. A. Houton, G. M. Burslem and A. J. Wilson, Chem. Sci., 2015, 6, 2382-2388.

36 S. Sami, E. Yildirim, M. Yurtsever, E. Yurtsever, E. Yilgor, I. Yilgor and G. L. Wilkes, Polymer, 2014, 55, 4563-4576.

37 D. H. Merino, A. T. Slark, H. M. Colquhoun, W. Hayes and I. W. Hamley, Polym. Chem., 2010, 1, 1263-1271.

38 P. J. Woodward, D. H. Merino, B. W. Greenland, I. W. Hamley, Z. Light, A. T. Slark and W. Hayes, Macromolecules, 2010, 43, 2512-2517.

39 D. Hermida-Merino, G. E. Newby, I. W. Hamley, W. Hayes and A. Slark, Soft Matter, 2015, 11, 5799-5803.

40 T. S. Babra, M. Wood, J. S. Godleman, S. Salimi, C. Warriner, N. Bazin, C. R. Siviour, I. W. Hamley, W. Hayes and B. W. Greenland, Eur. Polym. J., 2019, 119, 260-271.

41 J. P. Sheth, D. B. Klinedinst, G. L. Wilkes, I. Yilgor and E. Yilgor, Polymer, 2005, 46, 7317-7322.

42 T. Mondal, J. Sarkar and S. Ghosh, Chem. - Eur. J., 2016, 22, 10930-10936.

43 Q. Wan, M. Liu, L. Mao, R. Jiang, D. Xu, H. Huang, Y. Dai, F. Deng, X. Zhang and Y. Wei, Mater. Sci. Eng., C, 2017, 72, 352-358.

44 J. A. Sáez, B. Escuder and J. F. Miravet, Chem. Commun., 2010, 46, 7996-7998.

45 P. Maiti, G. Radhakrishnan, P. Aruna and G. Ghosh, Macromol. Symp., 2006, 241, 51-59.

46 M.-T. Leiendecker, C. J. Licht, J. Borghs, D. J. Mooney, M. Zimmermann and A. Böker, Macromol. Rapid Commun., 2018, 39, 1700711.

47 M. R. Molla and S. Ghosh, Chem. - Eur. J., 2012, 18, 9860-9869.

48 D. R. Canchi, D. Paschek and A. E. García, J. Am. Chem. Soc., 2010, 132, 2338-2344.
49 X.-M. Jiang, X.-J. Huang, S.-S. Song, X.-Q. Ma, Y.-M. Zhang, H. Yao, T.-B. Wei and Q. Lin, Polym. Chem., 2018, 9, 4625-4630.

50 Q. Lin, X.-M. Jiang, X.-Q. Ma, J. Liu, H. Yao, Y.-M. Zhang and T.-B. Wei, Sens. Actuators, B, 2018, 272, 139-145.

51 W. Dong, H. Wu, M. Chen, Y. Shi, J. Sun, A. Qin and B. Z. Tang, Polym. Chem., 2016, 7, 5835-5839.

52 G. Yang, H. Zhang, Y. Wang, X. Liu, Z. Luo and J. Yao, Sens. Actuators, B, 2017, 251, 773-780.

53 Q. Lin, B. Sun, Q.-P. Yang, Y.-P. Fu, X. Zhu, T.-B. Wei and Y.-M. Zhang, Chem. - Eur. J., 2014, 20, 11457-11462.

54 D. Dai, Z. Li, J. Yang, C. Wang, J.-R. Wu, Y. Wang, D. Zhang and Y.-W. Yang, J. Am. Chem. Soc., 2019, 141, 4756-4763.

55 S. K. Sahoo, D. Sharma, R. K. Bera, G. Crisponi and J. F. Callan, Chem. Soc. Rev., 2012, 41, 7195-7227.

56 Y.-M. Zhang, W. Zhu, X.-J. Huang, W.-J. Qu, J.-X. He, H. Fang, H. Yao, T.-B. Wei and Q. Lin, ACS Sustainable Chem. Eng., 2018, 6, 16597-16606.

57 Y.-M. Zhang, J.-X. He, W. Zhu, Y.-F. Li, H. Fang, H. Yao, T.-B. Wei and Q. Lin, Mater. Sci. Eng., C, 2019, 100, 62-69.

58 H. Xie, F. Zeng, C. Yu and S. Wu, Polym. Chem., 2013, 4, 5416-5424.

59 H. Wan, P. Gu, F. Zhou, H. Wang, J. Jiang, D. Chen, Q. Xu and J. Lu, Polym. Chem., 2018, 9, 3893-3899.

60 G. G. V. Kumar, M. P. Kesavan, G. Sivaraman and J. Rajesh, Sens. Actuators, B, 2018, 255, 3194-3206.

61 Q. Lin, P.-P. Mao, Y.-Q. Fan, L. Liu, J. Liu, Y.-M. Zhang, H. Yao and T.-B. Wei, Soft Matter, 2017, 13, 7085-7089.

62 W. A. Wan Ibrahim, L. I. Abd Ali, A. Sulaiman, M. M. Sanagi and H. Y. Aboul-Enein, Crit. Rev. Anal. Chem., 2014, 44, 233-254.

63 M. Kataria, M. Kumar and V. Bhalla, J. Indian Chem. Soc., 2018, 95, 1559-1577.

64 J. Liu, Y.-Q. Fan, S.-S. Song, G.-F. Gong, J. Wang, X.-W. Guan, H. Yao, Y.-M. Zhang, T.-B. Wei and Q. Lin, ACS Sustainable Chem. Eng., 2019, 7, 11999-12007. 